\title{
DNA Methylation and Asthma Acquisition During and Post-Adolescence, an Epigenome-Wide Longitudinal Study.
}

\section{Aniruddha Rathod}

University of Memphis https://orcid.org/0000-0002-4921-0952

\section{Hongmei Zhang ( $\boldsymbol{V}$ hzhang6@memphis.edu )}

University of Memphis https://orcid.org/0000-0003-3557-0364

\section{Hasan Arshad}

University of Southampton Faculty of Medicine

\section{Susan Ewart}

Michigan State University College of Veterinary Medicine

\section{Caroline Relton}

University of Bristol Medical School

\section{Wilfried Karmaus}

The University of Memphis School of Public Health

\section{John Holloway}

University of Southampton Faculty of Medicine

\section{Research}

Keywords: DNA Methylation, IOWBC, ALSPAC, Asthma Transition, Sex-specificity

Posted Date: August 5th, 2021

DOI: https://doi.org/10.21203/rs.3.rs-757593/v1

License: (ㅇ) (1) This work is licensed under a Creative Commons Attribution 4.0 International License. Read Full License

Version of Record: A version of this preprint was published at Journal of Personalized Medicine on February 2nd, 2022. See the published version at https://doi.org/10.3390/jpm12020202. 


\section{Abstract}

Background- While the majority of asthma starts in early childhood, asthma onset in some individuals occurs during adolescence or in adulthood. However, the pathogenesis of later onset asthma as well as the observed sex specificity are not well understood.

Objective We hypothesized that DNAm at specific CpG sites measured before disease onset, either in pre- or post-adolescence would be associated with asthma acquisition both during adolescence and in later adulthood.

Methods- Subjects from the Isle of Wight Birth Cohort (IOWBC) were included. DNAm in blood at ages 10 (preadolescence) and 18 (post-adolescence), and asthma acquisition from age 10-18, and 18-26 years was studied. To improve statistical power, we first screened epigenome-wide CpGs based on the association of DNAm at 10 years with asthma acquisition from 10-18 years. Logistic regression with repeated measures were then applied to the $\mathrm{CpGs}$ that survived screening to examine the associations of pre-adolescence DNAm with asthma acquisition from pre-to post-adolescence, and post-adolescence DNAm with asthma acquisition from postadolescence to adulthood. The effect of DNAm on asthma acquisition at different transition period was evaluated using interaction terms. The ALSPAC birth cohort was used for independent replication. For biological assessment of identified CpGs, pathway enrichment analysis and Differentially Methylated Regions were assessed.

Results- Significant interaction effects of DNAm and transition period (10-18 or 18-26 years) on asthma acquisition were found for $17 \mathrm{CpGs}$ in males and $98 \mathrm{CpGs}$ in females (FDR=0.05) in IOWBC. Consistent interaction effects were observed for $9 \mathrm{CpGs}$ in males and $53 \mathrm{CpGs}$ in females in ALSPAC. For CpGs not showing interaction effects (i.e., effect of DNAm is stable over time), association with asthma acquisition was found for $38 \mathrm{CpGs}$ in males and $52 \mathrm{CpGs}$ in females in IOWBC. Of these $90 \mathrm{CpGs}$, at $13 \mathrm{CpG}$ in males and 37 CpG in females, consistent direction of associations was observed in ALSPAC. Genes that the identified CpGs were mapped to, e.g., AKAP1 and ENO1, have shown to be associated with asthma.

Conclusion- DNAm at specific CpGs is associated with asthma acquisition and such association is likely to be sex and transition period specific.

\section{Introduction}

Asthma is the most prevalent chronic respiratory condition ${ }^{1}$ affecting $1-18 \%$ of population in several countries $^{2}$. Over recent decades, childhood asthma has become a major public health issue ${ }^{3}$ with an increasing prevalence worldwide ${ }^{4}$. Environmental factors such as air pollution, infectious agents, and tobacco smoke have been shown to be associated with the development of asthma ${ }^{5}$.

DNA methylation (DNAm), a robust and stable epigenetic mark, represents a potential mechanism for environmental impact on human diseases ${ }^{6}$. Recent studies suggest that DNAm signatures of cytosinephosphate-guanines $(\mathrm{CpG})$ sites are associated with asthma ${ }^{7-9}$. Since peripheral blood is readily obtainable and easy to handle in laboratory processing, and information of immune cells in blood is relevant to asthma 
pathogenesis ${ }^{10}$, DNAm in peripheral blood cells has been commonly examined in epigenome-wide studies of asthma $^{11-14}$.

While the development of asthma clearly reflects the combination of inherited susceptibility and environmental exposures, the pathogenesis and underlying biological mechanisms involved in the onset of asthma later in life are not well understood. Asthma most commonly develops during early childhood ${ }^{15}$, and the prevalence of asthma depends on gender and age. Asthma is more prevalent among pre-adolescent boys, while it becomes more prevalent among females after puberty with prevalence in males and females being approximately equal in adulthood ${ }^{16-18}$. However, the pathogenesis of these sex differences in asthma across adolescence and adulthood remain unclear.

Although previous studies have demonstrated association between DNAm and asthma, the role that DNAm plays in asthma acquisition, especially during the critical transition period from pre- to post- adolescence, and how its role changes over time, e.g., from adolescence to adulthood, are unknown. Findings from this type of studies will not only identify important markers for asthma acquisition, and more importantly, benefit our future efforts in asthma prediction and consequently asthma prevention. To this end, in this study, for each gender, we examined the association between DNAm at pre-adolescence and asthma acquisition from pre- to postadolescence (10 to 18 years), and between DNAm at post-adolescence and asthma acquisition from postadolescence to adulthood (18-26 years), utilizing genome-wide DNA methylation data. We hypothesized that DNAm at specific $\mathrm{CpG}$ sites measured before disease onset, either in pre- or post-adolescence would be associated with asthma acquisition both during adolescence and in later adulthood and that there would be differences in such DNA methylation patterns by time window (adolescence or post-adolescence) and by gender.

\section{Methods}

\section{Study population}

The study population comprised of children born between January 1, 1989 and February 28, 1990 on the Isle of Wight (IoW), UK (IOWBC) ${ }^{19}$. Out of the 1,536 children born and recruited, 1,456 in IOWBC were available for further follow-up at ages 1, 2, 4, 10, 18 and 26 years. Ethics approval was obtained by the local research ethics committee (NRES Committee South Central - Hampshire B) $\left(06 /\right.$ Q1701/34) ${ }^{20}$.

\section{Asthma acquisition}

Questionnaires that included the questions of the International Study of Asthma and Allergy in Childhood (ISAAC) was filled by parents/participants at ages 4, 10, 18 and 26 years ${ }^{8,21-23}$. Asthma was defined as "physician diagnosed asthma" and "wheezing or whistling in the chest in the last 12 months" or "current treatment for asthma." Subjects with asthma at age four years were excluded. The outcome used in this study, asthma acquisition, was defined as individuals who were asthma free at age 10 years and recorded as having asthma at age 18 years (no $\rightarrow$ yes). The same definition was applied for asthma acquisition from 18 to 26 years (no $\rightarrow$ yes). Subjects who did not have asthma at both the transition periods were taken as reference (no $\rightarrow$ no).

\section{DNA methylation}


Using a standardized salting procedure, DNA was extracted from peripheral whole blood samples collected at ages 10 and 18 years $^{24}$. Fluorometric quantitation was used to estimate DNA concentration. Methylation levels at each CpG site was measured using Illumina Infinium HumanMethylation450 or MethylationEPIC BeadChips (Illumina, Inc., San Diego, ca, USA). Probes that did not reach a p-value of $10^{-16}$ in at least $95 \%$ of samples were exclude. The same criterion was applied to exclude samples, i.e., samples with p-value $>10^{-16}$ in at least $95 \%$ of the CpGs. CpGs on sex chromosome were excluded.

Using CPACOR pipeline, DNA methylation (DNAm) was pre-processed for the data from both HumanMethylation450 and MethylationEPIC. DNAm intensities were quantile normalized using the minfi R computing package ${ }^{25}$. The quantile normalized intensities at autosomal probes were then converted to beta values. Principal components (PCs) inferred based on control probes were used to represent latent chip to chip and technical variation. We determined PCs based on DNAm at shared control probes of the two DNAm platforms HumanMethylation450 and MethylationEPIC. In total, 195 shared control probes were used to calculate the control probe PCs with top 15 PCs included in our study to represent latent batch factors ${ }^{26}$. In this study, CpG sites common between Illumina 450k platform and EPIC platform were examined. In additions, CpG sites were excluded if the minor allele frequency of a probe SNP at that site is $>0.7 \%$ (i.e., $\geq 10$ out of 1456 subjects expected to have the minor allele in the cohort) and the probe SNP was within 10 base pairs of the targeted CpG site. After quality control and pre-processing, 442,475 CpG sites were included in subsequent analysis.

Since whole blood is a mixture of distinct cell types ${ }^{27}$, there is a need to adjust for cell type composition to account for their potentially confounding effects ${ }^{28}$. Cell type proportions were estimated using the Bioconductor minfi package 2930 . The estimated cell type proportions of CD4 + T cells, natural killer cells, neutrophil, B cells, monocytes, and eosinophil cells were included in the analyses as confounders.

\section{Covariates}

Atopic status was evaluated at ages 10 and 18 years based on results from skin prick test (SPT) on 11 common allergens (house dust mite, cat dander, dog dander, grass pollen mix, tree pollen mix, Alternaria alternate, Cladosporium herbarium, cow's milk, hen's egg, peanut, and cod). Being SPT positive to one or more of the 11 allergens was treated as being atopic. Active smoking status at 18 and 26 years was recorded as 'yes' if the participant was a current smoker at that respective age. Second-hand smoke exposure was coded at age 18and 26-years using information obtained from smoking status of parents and other smokers in the household. To evaluate the contribution of transition periods, 10-18 and 18-26 years, to the association of DNAm with asthma transition, transition periods were included in the analyses as adjusting factors.

\section{Statistical analyses}

By regressing the M-values (base-2 logit transformed beta values of DNAm) at each CpG site on the aforementioned 15 PCs and 6 cell type proportions, we obtained cell-type and batch-adjusted DNAm (residuals) at each of the 442,475 CpG sites for each gender in IOWBC. Screening of CpG sites was done to obtain DNAm potentially associated with asthma acquisition from pre- to post-adolescence using simple linear regressions. Here, asthma acquisition from 10 to 18 years of age was the independent variable and DNAm at age 10 years was the dependent variable. The analysis was stratified by gender. For the screening purpose, multiple testing 
was adjusted by controlling false discovery rate (FDR) at a higher rate of 0.2 . $\mathrm{CpG}$ sites that passed screening were included in subsequent analyses.

Logistic regressions with repeated measurements were applied to the $\mathrm{CpGs}$ that passed screening to evaluate the association of asthma acquisitions (no $\rightarrow$ yes) at two transition periods (10-18 years and 18-26 years) with DNAm at earlier ages (10 and 18 years, respectively). The use of repeated measures allowed us to achieve a higher statistical power and detect the desired effect size. This approach is especially desired in the situation of small sample sizes. Along with other covariates- atopic status at 10 and 18 years, active and second-hand smoking status at 18 and 26 years, to assess whether the associations are different at different transition periods, in addition to the main effects of DNAm, we also tested interaction effects between DNAm and transition period. For both situations (the models with main effects only, and the models that included interaction effects) multiple testing was adjusted by controlling FDR of 0.05 .

\section{Replication cohort - ALSPAC}

In addition to the utilization of repeated measures in our statistical modeling, to further assess the informativity of findings in IOWBC, an independent replication cohort, the Avon Longitudinal study of Parents and Children (ALPSAC) cohort ${ }^{31-33}$, was included to examine $\mathrm{CpGs}$ showing significant interaction effects with transition periods in IOWBC. DNAm in the ALSPAC cohort was assessed using the Infinium HumanMethylation450 BeadChip. DNAm data on 604 children in the ALSPAC cohort were available at ages 7 and 17 years ${ }^{34}$. DNAm pre-processing was performed by correcting for batch effects using the minfi package ${ }^{25}$ and removing CpGs with detection $p$-value $\geq 0.01$. Samples were flagged that contained sex-mismatch based on $X$-chromosome methylation. Estimated cell type proportions of CD4 + T cells, natural killer cells, neutrophil, B cells, monocytes, and granulocytes cells were used in the analyses to adjust for cell heterogeneity.

Asthma acquisition status from 7 to 17 years, and 17 to 22 years was included in the analysis. It was defined as having no asthma at age 7 years and having asthma at age 17 years. The same definition was applied for asthma acquisition from 17 to 22 years. Logistic regression with repeated measurements were used with similar covariates (as those in IOWBC) available in ALSPAC, i.e., atopy status at age 7 years and second-hand smoke exposure status at age 17 and 24 years. Please note that the study website contains details of all the data that is available through a fully searchable data dictionary and variable search tool (http://www.bristol.ac.uk/alspac/researchers/our-data/).

\section{Detection of differentially methylated regions (DMR)}

Differentially methylated regions (DMRs) were identified using the DMRcate package in $\mathrm{R}^{35}$. To secure a sufficient number of CpGs for DMR enrichment analysis and to avoid missing important DMRs, CpGs with DNAm (in $M$ values) associated with asthma acquisition via logistic regression at FDR of 0.4 were included in the analysis.

\section{Pathway enrichment analyses}

For CpGs showing associations of DNAm with asthma acquisition status, the genes annotated to the CpGs were summarized along with information such as gene location, chromosome number based on Illumina's manifest file and USCS genome browser (https://genome.ucsc.edu). Pathway enrichment analysis of the identified CpGs was conducted using the gometh function ${ }^{36}$ in the R package to better understand their biological functionality. 


\section{Results}

Since our study focused on asthma acquisition starting from age 10 years in IOWBC, subjects with asthma at four years were excluded. Participants in IOWBC with both asthma transition and DNAm data available at ages 10 and 18 years were included in the study. The subsamples represented the complete IOWBC (such that no asthma at age 4 years) with respect to asthma acquisition, active and second-hand smoking, and atopy status, (Table 1a \& 1b).

In total, $55 \mathrm{CpGs}$ for males and $183 \mathrm{CpGs}$ for females in IOWBC passed screening based on their potential associations with asthma acquisition from 10 to 18 years of age. These $\mathrm{CpGs}$ were included in subsequent analyses for their longitudinal associations of DNAm with asthma acquisition from pre to post-adolescence and from post-adolescence to young adulthood and for interaction effects between DNAm and transition periods, using logistic regressions with repeated measurements. The utilization of logistic regressions with repeated measures allowed us to identify effect sizes with a higher statistical power even if the number of "cases" (asthma acquisitions) was relatively small.

After adjusting for multiple testing by controlling FDR of 0.05 , statistically significant interaction effects of DNAm and transition period were observed at $17 \mathrm{CpGs}$ in males and $98 \mathrm{CpGs}$ in females (no common CpGs identified between males and females), controlling for atopy status, active and second-hand smoking (Figures 1 and 2, Supplemental table 1). Of the 17 identified CpGs in males, $4 \mathrm{CpGs}(23.5 \%)$ were located in the promoter region, while for the $98 \mathrm{CpGs}$ identified in females, a much larger portion of CpGs (54 CpGs, $55.1 \%$ ) were in the promoter region. For 7 of the $17 \mathrm{CpGs}$ in males, an increase in DNAm is associated with an increased odds of asthma acquisition in the 10-18 transition period, but decreased odds in the 18-26 period (Figures $1 \mathrm{a}$ and $1 \mathrm{~b}$, Supplemental table 1a). For 47 of the $98 \mathrm{CpGs}$ in females, an increase in DNAm is associated with a decreased odds of asthma acquisition in the 10-18 transition period, but increased odds in the 18-26 period (Figures 2a and $2 b$, Supplemental table $1 \mathrm{~b}$ ). In addition, the overall effect sizes at the first transition period was larger than the effect sizes in the second transition period.

For CpGs not showing significant interaction effects between DNAm and transition period, we assessed the main effects of DNAm on asthma acquisition via logistic regression models with repeated measures. After adjusting for multiple testing at FDR=0.05, we identified $38 \mathrm{CpGs}$ in males and $52 \mathrm{CpGs}$ in females (Figure 3 , Supplemental table 2) showing association of DNAm with asthma acquisition status (no common CpGs between males and females). Of the $38 \mathrm{CpGs}$ in males, $13 \mathrm{CpGs}(34.2 \%)$ were in the promoter region, while for the $52 \mathrm{CpGs}$ identified in females, a much larger portion of CpGs (25 CpGs, 48.1\%) were in the promoter region. At 25 of the $38 \mathrm{CpGs}$ in males, an increase in DNAm is associated with a decreased odds of asthma acquisition (Supplemental table 2a). At 45 of the $52 \mathrm{CpGs}$ in females, an increase in DNAm is associated with a decreased odds of asthma acquisition (Supplemental table 2b). Overall, the effect sizes of DNAm on asthma acquisition were larger in males than in females.

Altogether, we identified $115 \mathrm{CpGs}$ (17 in males) showing interactions with transition period and $90 \mathrm{CpGs}$ (38 in males) showing main effects (excluded CpGs with interaction effects), leading to a total of 205 identified CpGs. We further tested these CpGs in the ALSPAC cohort. For the $115 \mathrm{CpGs}$ (17 in males) showing interaction effects in IOWBC, consistent directions of interaction effects were observed at $9 \mathrm{CpGs}$ in males, with $1 \mathrm{CpG}$ being statistically significant, and $53 \mathrm{CpGs}$ in females, with $3 \mathrm{CpGs}$ being statistically significant, compared to the 
directions of associations identified in the IOWBC (Supplemental tables 1a and 1b). Of the 9 CpGs showing consistent interactions in males, $2 \mathrm{CpGs}(22.2 \%)$ were in the body region of gene, while of the 53 such CpGs in females, $34(64.2 \%)$ were in the promoter region. For the $90 \mathrm{CpGs}$ (38 in males) showing main effects on asthma acquisition, $13 \mathrm{CpGs}$ in males (3 CpGs [23.1\%] in the promoter region) and $37 \mathrm{CpGs}$ in females (19 CpGs [51.4\%] in the promoter region) showed consistent directions of main effects (Supplemental table 2a and 2b) between the two cohorts. The flowchart of the study along with brief summaries of results is in Figure 4 .

Pathway enrichment analyses was conducted based on IOWBC-discovered CpGs for each sex (55 in males and 150 in females with $205 \mathrm{CpGs}$ in total) to better understand their biological functionality. These CpGs were mapped to 54 and 149 genes in males and females respectively. Using these CpGs in the gometh function in R, we identified 212 biological processes in males and 228 in females that were enriched at $p$-value of 0.05 . Although none of the biological processes survived multiple testing at FDR of 0.05 , genes involved in the top processes for each sex based on statistical significance (top 10 processes in Table 2) were potentially important and may deserve a further assessment. For males, multiple biological processes among the top 10 for males focus on catabolic processes (breakdown glucose for energy), while for females they are biosynthetic processes (synthesizing glucose from food). Among these top processes identified for males, 13 genes corresponding to the identified $\mathrm{CpGs}$ were involved in those processes, and for females, 60 genes were involved (Supplemental table 3). Of the 13 genes, CpGs on five ( 39\%) genes showing consistent associations (interaction or main effects) between IOWBC and ALSPAC, and of the 60 genes, 34 ( 57\%) genes shown such consistency between the two cohorts.

For DMR enrichment analysis, we used CpGs in the screening process that were statistically significant at FDR of 0.4 to cover epigenetic information comprehensively on asthma acquisition. In total, $427 \mathrm{CpGs}$ in males and $372 \mathrm{CpGs}$ in females were included in the analysis. We identified three DMRs in males and three DMRs in females (Table 3).

\section{Discussion}

We assessed the longitudinal association of DNAm measured at earlier ages with asthma acquisition at later ages for each sex based on data in two independent cohorts with IOWBC as the discovery cohort and ALSPAC as the replication cohort. In the IOWBC, at $205 \mathrm{CpGs}$, pre-adolescence DNAm was shown to be associated with the odds of asthma acquisition from pre- to post-adolescence, and post-adolescence DNAm was associated with asthma acquisition from post-adolescence to adulthood. At 112 of these $205 \mathrm{CpGs}$ (54.6\%), consistent associations were observed in the ALSPAC cohort, including statistically significant findings at $7 \mathrm{CpGs}$. These $112 \mathrm{CpGs}$ included $62 \mathrm{CpGs}$ (9 in males) showing transition-specific associations with asthma acquisition in that the association of DNAm with asthma acquisition at these $62 \mathrm{CpGs}$ was different between the pre- to postadolescence transition period and the post- to adulthood transition period.

Our findings also indicated significant differences between males and females. For the $62 \mathrm{CpGs}$ showing consistent transition-specific effects between the two cohorts, at most of the CpGs in males, we found that an increase in DNAm was associated with an increased odds of asthma acquisition during the period from pre-to post-adolescence transition, while for the next transition period, at most of the CpGs, increased DNAm was associated with decreased odds. However, in females, at most of the $\mathrm{CpGs}$, the associations were opposite compared to those in males; in females, an increase in DNAm was shown to be associated with a decreased 
odds of asthma acquisition from pre- to post-adolescence at most CpGs, but with increased odds at most of the $\mathrm{CpGs}$ in the transition period from post-adolescence to adulthood. Among the $50 \mathrm{CpGs}$ (13 in males) showing main effects on asthma acquisition, although at most of the $\mathrm{CpGs}$, an increase in DNAm was associated with a decreased odds of asthma acquisition for both males and females, the proportion of such CpGs was larger in females than in males. Furthermore, the effect sizes were overall weaker in females than in males. Before adolescence, asthma is more prevalent in males but during adolescence, more females acquire asthma and the prevalence of asthma in females surpasses that of males. The unique CpGs identified for each sex without any overlap and the inconsistent associations of DNAm with asthma acquisition between males and females seemed to be related to the gender-reversal phenomenon of asthma prevalence from pre- to post-adolescence.

Although we did not identify statistically significant biological processes after adjusting for multiple testing, biological processes involved in host immune function related to IL7 (i.e., interleukin-7-mediated signaling pathway, response to interleukin-7, cellular response to interleukin-7) were among the top processes determined based on statistical significance. IRS1 gene was involved in the processes related to IL-7 and its mapped CpG (cg11620807) showed consistent association between the two cohorts. IL7 signaling has been suggested to promote immunopathogenesis of asthma ${ }^{37,38}$, indicating the potentially informativity of the identified CpGs on asthma acquisition. In addition, the TMEM194A gene identified based on DMR analyses in males has been previously shown to be associated with asthma in GWAS catalog ${ }^{39}$. For females, gene SERPINE2 in one of the identified DMRs has been connected with asthma based on genetic studies ${ }^{40}$.

The gene $A K A P 1$, mapped to cg02467794, showing consistent and statistically significant interaction effects in both cohorts in females, has been showed to be associated with asthma in the Agricultural Lung Health Study ${ }^{41}$. Although there was no overlap in identified $\mathrm{CpGs}$ between males and females, the gene ENO1 was among the mapped genes of the IOWBC-discovered CpGs in both sexes. The detection of IgG autoantibodies to alpha-enolase has been shown to be the most significant indicator for distinguishing severe asthma from mildto-moderate asthma $(\mathrm{OR}=5.2,95 \% \mathrm{Cl}=2.1-12.9$, $\mathrm{p}$-value $<.001)$. It has been shown that alpha-enolase, an autoantigen, was associated with severe asthma ${ }^{42}$. The connection of gene ENO1 with asthma acquisition shown in our study is consistent with its differentiation between severe and mild-to-moderate asthma. Further assessment on CpGs located on this gene is likely to benefit the potential of the $\mathrm{CpGs}$ as epigenetic markers for asthma acquisition.

The strength of this study exists in its focus on longitudinal assessment of asthma acquisition at two important transition periods, pre- to post-adolescence and to young adulthood, along with DNAm at two critical time points, pre- and post-adolescence. To our knowledge, this is the first study to examine the epigenetics of asthma acquisition from pre- to post- adolescence, and post-adolescence to young adulthood with respect to gender and transition period specificity.

Although for the CpGs discovered in IOWBC, more than $50 \%$ showed consistent findings in ALSPAC, statistical significance was observed at a small number of CpG sites. One reason for this lack of significance might be the age differences between the two cohorts. In addition, there is a potential concern of data double dipping. However, we do not see this as a significant concern in that the statistical model applied in the screening process (linear regression without covariates such as atopic and smoking status) was different from the model in the final analyses (logistic regression with potential covariates). We also noticed that the number of asthma 
acquisitions at each age was relatively small. However, our utilization of repeated measures in regression analyses had a potential to ease this concern, and the inclusion of the ALSPAC replication cohort to further examine the IOWBC-findings with focus on consistent direction of associations further relieved our worries to a certain extent. Another potential limitation is in the design of data analyses, which focused on each individual $\mathrm{CpG}$ site. However, $\mathrm{CpG}$ sites might be correlated and work jointly to impact the risk of asthma acquisition, which certainly deserves future investigations accompanied by carefully designed analytical plans. Finally, both cohorts, although independent, are mainly Caucasians. Thus, our findings are likely limited to only this population. Nevertheless, the identified $\mathrm{CpGs}$ based on two independent cohorts have a potential to guide future studies in asthma acquisition prediction at different transition periods.

\section{Abbreviations}

CpG: 5'-C-Phosphate-G-3'

DNAm: Deoxyribonucleic acid methylation

DMRs: Differentially Methylated Regions

FDR: False Discovery Rate.

\section{Declarations}

\section{Ethics approval and consent to participate}

Isle of Wight Local Research Ethics Committee approved 10 years assessment as well as collection of blood for genetic studies into asthma and allergy (No. 18/98, dated 07/20/1998). For the 18-year follow-up, ethical approval was given by the Isle of Wight, Portsmouth and SE Hampshire Local Research Ethics Committee (No. 06/Q1701/34, dated 06/16/2006). The Internal Review Board (IRB) at the University of Memphis (FWA00006815, IRB ID: \#3917) gave approval for data analysis at University of Memphis. Written informed consent was obtained from parents during in-person visits. For participants assessed by phone interview, consent was documented on the consent form with the name of the person giving consent, and the name and signature of the person taking the form were recorded.

For ALSPAC, ethical approval for the study was obtained from the ALSPAC Ethics and Law Committee and the Local Research Ethics Committees. Consent for collection of biological samples was provided in accordance with the Human Tissue Act (2004). Informed consent for the use of data collected via questionnaires and clinics was obtained from participants following the recommendations of the ALSPAC Ethics and Law Committee at the time. For age seven years, United Bristol Healthcare Trust: E4168 (ALSPAC Hands on Assessments at Age Seven), Southmead Health Services: 67/98 (Avon Longitudinal Study of Pregnancy and Childhood (ALSPAC) Hands on Assessments at Age Seven) and Frenchay Healthcare Trust: 98/52 (Avon Longitudinal Study of Pregnancy and Childhood (ALSPAC). Hands on Assessments at Age Seven). For age 15 years, Central \& South Bristol Research Ethics Committee (UBHT): 06/Q2006/53 Avon Longitudinal Study of Parents and Children (ALSPAC), Hands on Assessments: Teen Focus 3 (Focus 15+), and for age 17 years, North Somerset \& South Bristol Research Ethics Committee: 08/H0106/9 Avon Longitudinal Study of Parents and Children (ALSPAC), 
Hands on Assessments: Teen Focus 4 (Focus 17+). Full details of ethical approvals (local committees and approval numbers) are available at

http://www.bristol.ac.uk/media-

library/sites/alspac/documents/governance/Research\%20Ethics\%20Committee\%20approval\%20references.pdf

Consent for publication- Not applicable

\section{Data availability statement}

The datasets used and/or analyzed during the current study are available from the corresponding author on reasonable request. For the ALSPAC data, please contact the ALSPAC executive committee (alspacexec@bristol.ac.uk).

\section{Conflicts of interest}

The authors declare that they have no conflicts of interest.

\section{Funding}

This study was supported by the National Institutes of Health research fund R01Al121226 (MPI: H Zhang and JW Holloway). Part of the methylation data generation was supported by R01AI091905 (PI: W Karmaus). The Isle of Wight Birth Cohort assessments have been supported by the National Institutes of Health USA (Grant no. R01 HL082925, H. Arshad), Asthma UK (Grant no. 364. S.H. Arshad) and the David Hide Asthma and Allergy Research Trust. None of the sponsors were involved in study design, collection, analysis, and interpretation of data, the writing of the manuscript, and the decision to submit the manuscript for publication. The authors are thankful to all the IOWBC participants and greatly appreciate the support of High-Performance Computing facility at the University of Memphis.

The UK Medical Research Council and Wellcome (Grant ref: 217065/Z/19/Z) and the University of Bristol provide core support for ALSPAC. A comprehensive list of grants funding is available on the ALSPAC website (http://www.bristol.ac.uk/alspac/external/documents/grant-acknowledgements.pdf). Generation of methylation array data was specifically funded by NIH R01Al121226, R01AI091905, BBSRC BBI025751/1 and BB/I025263/1, MRC MC_UU_12013/1, MC_UU_12013/2, MC_UU_12013/8.

\section{Statement of author contribution}

$\mathrm{HZ}$ designed the study, AR analyzed the data, AR and $\mathrm{HZ}$ interpreted findings and drafted the manuscript, WK, HA, CR, and JWH supervised the study, SE, JWH provided DNA methylation data, and all authors reviewed the manuscript.

\section{Acknowledgement}

The authors are thankful to the nurses and staff at the David Hide Asthma \& Allergy Research Centre, Isle of Wight, UK, for their help in recruitment and sample collections, and are thankful to all the cohort participants. Our special thanks also go to the High-Performance Computing facility provided by the University of Memphis. 
For ALSPAC, DNA extraction and generation of Illumina array data was carried out in the Bristol Bioresource Laboratories at the University of Bristol, UK. We are extremely grateful to all the families who took part in this study, the midwives for their help in recruiting them, and the whole ALSPAC team, which includes interviewers, computer and laboratory technicians, clerical workers, research scientists, volunteers, managers, receptionists and nurses.

\section{References}

1. Fuchs O, Bahmer T, Rabe KF, von Mutius E. Asthma transition from childhood into adulthood. The Lancet Respiratory medicine. 2017;5(3):224-234.

2. Asthma GIf. Global Strategy for Asthma management and prevention. 2020.

3. Castro-Rodriguez JA, Forno E, Rodriguez-Martinez CE, Celedón JC. Risk and Protective Factors for Childhood Asthma: What Is the Evidence? The journal of allergy and clinical immunology In practice. 2016;4(6):1111-1122.

4. Loftus PA, Wise SK. Epidemiology of asthma. Current opinion in otolaryngology \& head and neck surgery. 2016;24(3):245-249.

5. Murrison LB, Brandt EB, Myers JB, Hershey GKK. Environmental exposures and mechanisms in allergy and asthma development. The Journal of Clinical Investigation. 2019;129(4):1504-1515.

6. Joubert BR, Felix JF, Yousefi P, et al. DNA Methylation in Newborns and Maternal Smoking in Pregnancy: Genome-wide Consortium Meta-analysis. Am J Hum Genet. 2016;98(4):680-696.

7. DeVries A, Vercelli D. Early predictors of asthma and allergy in children: the role of epigenetics. Current opinion in allergy and clinical immunology. 2015;15(5):435-439.

8. Patel R, Solatikia F, Zhang H, et al. Sex-specific associations of asthma acquisition with changes in DNA methylation during adolescence. Clinical and experimental allergy : journal of the British Society for Allergy and Clinical Immunology. 2020.

9. Rathod A, Duan J, Zhang H, et al. Interweaving Between Genetic and Epigenetic Studies on Childhood Asthma. Epigenetics insights. 2020;13:2516865720923395.

10. Vercelli D. Does epigenetics play a role in human asthma? Allergology international : official journal of the Japanese Society of Allergology. 2016;65(2):123-126.

11. Reese SE, Xu CJ, den Dekker HT, et al. Epigenome-wide meta-analysis of DNA methylation and childhood asthma. The Journal of allergy and clinical immunology. 2019;143(6):2062-2074.

12. Arathimos R, Suderman M, Sharp GC, et al. Epigenome-wide association study of asthma and wheeze in childhood and adolescence. Clin Epigenetics. 2017;9:112.

13. Xu CJ, Soderhall C, Bustamante M, et al. DNA methylation in childhood asthma: an epigenome-wide metaanalysis. The Lancet Respiratory medicine. 2018;6(5):379-388. 
14. Rathod R, Zhang H, Karmaus W, et al. BMI trajectory in childhood is associated with asthma incidence at young adulthood mediated by DNA methylation. Allergy, asthma, and clinical immunology : official journal of the Canadian Society of Allergy and Clinical Immunology. 2021;17(1):77.

15. Martinez FD, Wright AL, Taussig LM, Holberg CJ, Halonen M, Morgan WJ. Asthma and wheezing in the first six years of life. The Group Health Medical Associates. N Engl J Med. 1995;332(3):133-138.

16. Koper I, Hufnagl K, Ehmann R. Gender aspects and influence of hormones on bronchial asthma - Secondary publication and update. The World Allergy Organization journal. 2017;10(1):46.

17. Pignataro FS, Bonini M, Forgione A, Melandri S, Usmani OS. Asthma and gender: The female lung. Pharmacological research. 2017;119:384-390.

18. Osman M, Hansell AL, Simpson CR, Hollowell J, Helms PJ. Gender-specific presentations for asthma, allergic rhinitis and eczema in primary care. Primary care respiratory journal : journal of the General Practice Airways Group. 2007;16(1):28-35.

19. Arshad SH, Holloway JW, Karmaus W, et al. Cohort Profile: The Isle Of Wight Whole Population Birth Cohort (IOWBC). International journal of epidemiology. 2018;47(4):1043-1044i.

20. Zhang H, Kaushal A, Merid SK, et al. DNA methylation and allergic sensitizations: A genome-scale longitudinal study during adolescence. Allergy. 2019;74(6):1166-1175.

21. Soto-Ramirez N, Arshad SH, Holloway JW, et al. The interaction of genetic variants and DNA methylation of the interleukin-4 receptor gene increase the risk of asthma at age 18 years. Clinical epigenetics. 2013;5(1):1.

22. Zhang H, Kaushal A, Soto-Ramirez N, et al. Acquisition, remission, and persistence of eczema, asthma, and rhinitis in children. Clinical and experimental allergy : journal of the British Society for Allergy and Clinical Immunology. 2018;48(5):568-576.

23. Ziyab AH, Karmaus W, Zhang H, et al. Association of filaggrin variants with asthma and rhinitis: is eczema or allergic sensitization status an effect modifier? International archives of allergy and immunology. 2014;164(4):308-318.

24. Zhang $\mathrm{H}$, Tong $\mathrm{X}$, Holloway JW, et al. The interplay of DNA methylation over time with Th2 pathway genetic variants on asthma risk and temporal asthma transition. Clin Epigenetics. 2014;6(1):8.

25. Aryee MJ, Jaffe AE, Corrada-Bravo H, et al. Minfi: a flexible and comprehensive Bioconductor package for the analysis of Infinium DNA methylation microarrays. Bioinformatics (Oxford, England). 2014;30(10):13631369.

26. Lehne B, Drong AW, Loh M, et al. A coherent approach for analysis of the Illumina HumanMethylation450 BeadChip improves data quality and performance in epigenome-wide association studies. Genome biology. 2015;16:37.

27. Reinius LE, Acevedo N, Joerink M, et al. Differential DNA methylation in purified human blood cells: implications for cell lineage and studies on disease susceptibility. PloS one. 2012;7(7):e41361. 
28. Koestler DC, Christensen B, Karagas MR, et al. Blood-based profiles of DNA methylation predict the underlying distribution of cell types: a validation analysis. Epigenetics. 2013;8(8):816-826.

29. Jaffe AE, Irizarry RA. Accounting for cellular heterogeneity is critical in epigenome-wide association studies. Genome biology. 2014;15(2):R31.

30. Houseman EA, Accomando WP, Koestler DC, et al. DNA methylation arrays as surrogate measures of cell mixture distribution. BMC Bioinformatics. 2012;13:86.

31. Boyd A, Golding J, Macleod J, et al. Cohort Profile: the 'children of the $90 s^{\prime}-$-the index offspring of the Avon Longitudinal Study of Parents and Children. International journal of epidemiology. 2013;42(1):111-127.

32. Fraser A, Macdonald-Wallis C, Tilling K, et al. Cohort Profile: the Avon Longitudinal Study of Parents and Children: ALSPAC mothers cohort. International journal of epidemiology. 2013;42(1):97-110.

33. Northstone K, Lewcock M, Groom A, et al. The Avon Longitudinal Study of Parents and Children (ALSPAC): an update on the enrolled sample of index children in 2019. Wellcome open research. 2019;4:51.

34. Relton CL, Gaunt T, McArdle W, et al. Data Resource Profile: Accessible Resource for Integrated Epigenomic Studies (ARIES). International journal of epidemiology. 2015;44(4):1181-1190.

35. Peters TJ, Buckley MJ, Statham AL, et al. De novo identification of differentially methylated regions in the human genome. Epigenetics \& chromatin. 2015;8:6.

36. Geeleher P, Hartnett L, Egan LJ, Golden A, Raja Ali RA, Seoighe C. Gene-set analysis is severely biased when applied to genome-wide methylation data. Bioinformatics (Oxford, England). 2013;29(15):1851-1857.

37. Reeder KM, Dunaway CW, Blackburn JP, et al. The common Y-chain cytokine IL-7 promotes immunopathogenesis during fungal asthma. Mucosal immunology. 2018;11(5):1352-1362.

38. Kelly EA, Koziol-White CJ, Clay KJ, et al. Potential contribution of IL-7 to allergen-induced eosinophilic airway inflammation in asthma. Journal of immunology (Baltimore, Md : 1950). 2009;182(3):1404-1410.

39. Hoang TT, Sikdar S, Xu CJ, et al. Epigenome-wide association study of DNA methylation and adult asthma in the Agricultural Lung Health Study. The European respiratory journal. 2020;56(3).

40. Himes BE, Klanderman B, Ziniti J, et al. Association of SERPINE2 with asthma. Chest. 2011;140(3):667-674.

41. Hoang TT, Sikdar S, Xu C-J, et al. Epigenome-Wide Association Study of DNA Methylation and Adult Asthma in the Agricultural Lung Health Study. European Respiratory Journal. 2020:2000217.

42. Nahm DH, Lee KH, Shin JY, Ye YM, Kang Y, Park HS. Identification of alpha-enolase as an autoantigen associated with severe asthma. The Journal of allergy and clinical immunology. 2006;118(2):376-381.

\section{Tables}

Due to technical limitations, table 1-3 is only available as a download in the Supplemental Files section. 

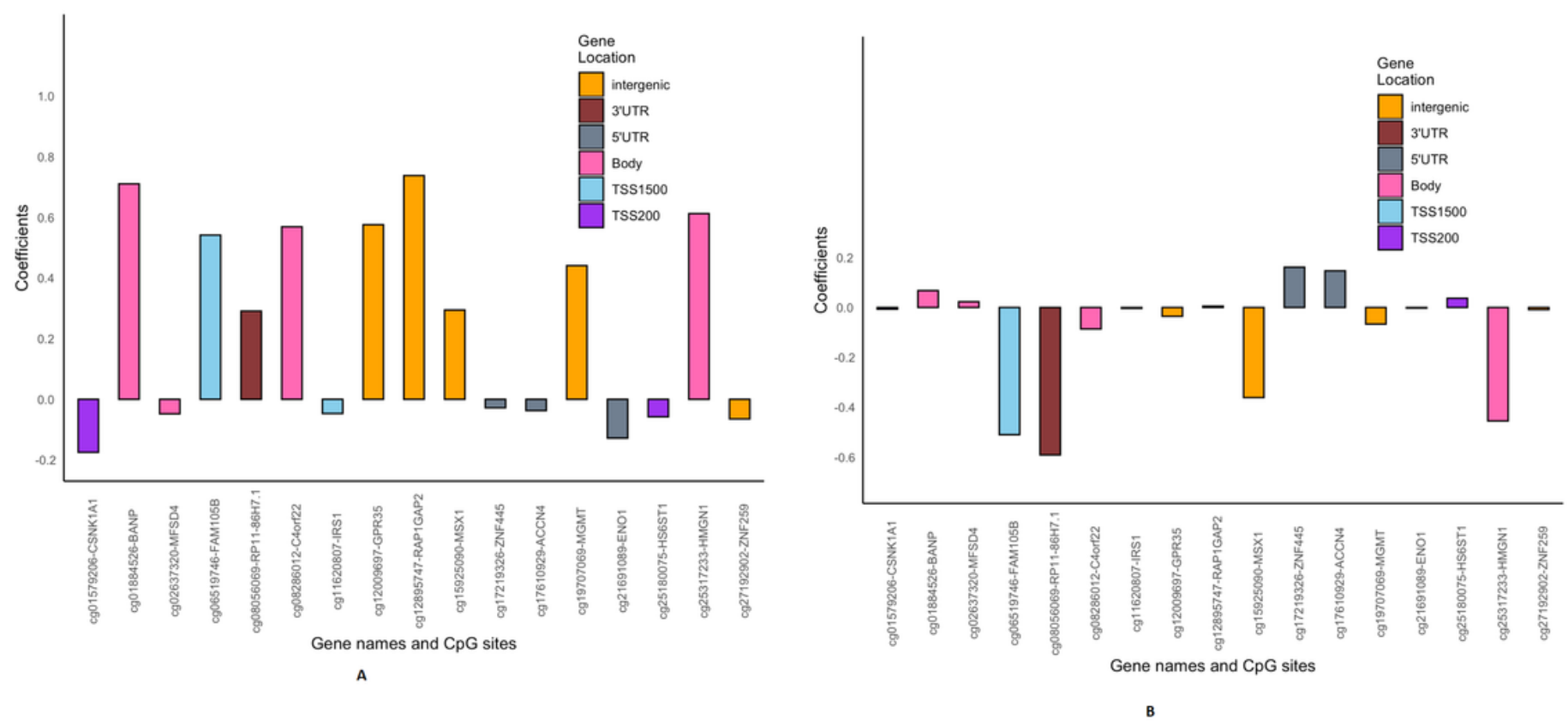

Figure 1

(A): Bar graph showing the direction of DNAm effect at each of the 17 identified CpGs in IOWBC from pre- to post-adolescence asthma acquisition in males. Gene names corresponding to each $\mathrm{CpG}$ site are also labeled on the X-axis. (B): Bar graph showing the direction of effect at each of the 17 identified CpGs in IOWBC from postadolescence to adulthood asthma acquisition in males. Gene names corresponding to each CpG site are also labeled on the X-axis.
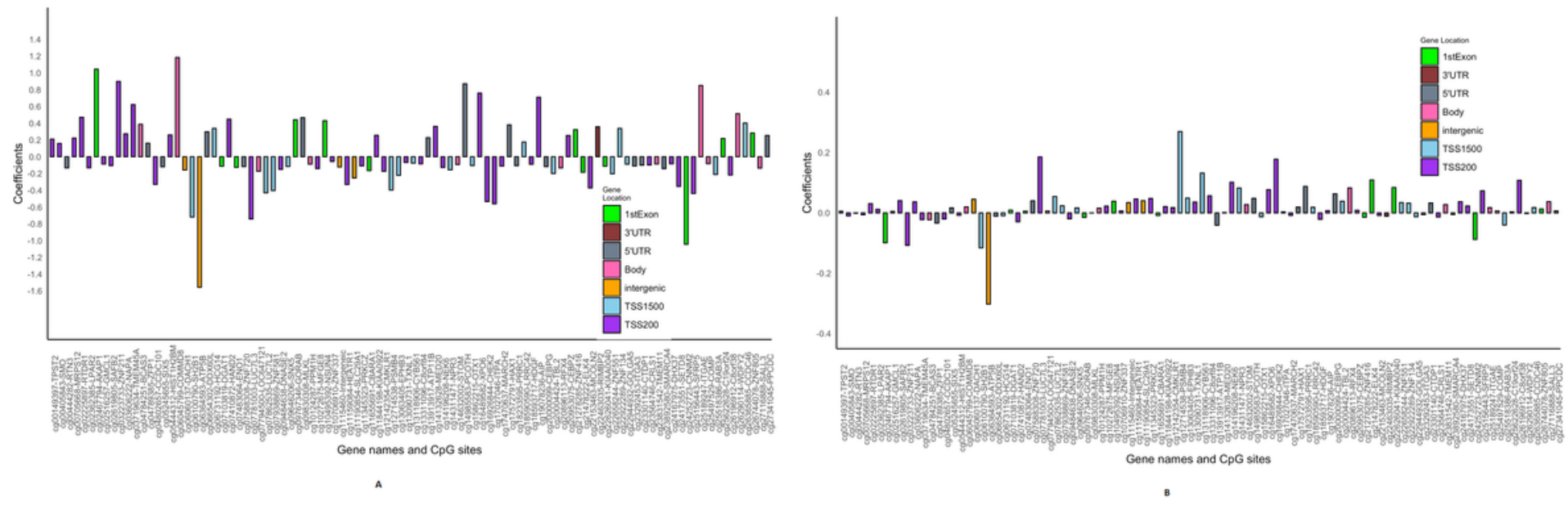

Figure 2

(A): Bar graph showing the direction of effect at each of the 98 identified CpGs in IOWBC from pre- to postadolescence asthma acquisition in females. Gene names corresponding to each $\mathrm{CpG}$ site are also labeled on the X-axis. (B): Bar graph showing the direction of effect at each of the 17 identified CpGs in IOWBC from post- 
adolescence to adulthood asthma acquisition in females. Gene names corresponding to each CpG site are also labeled on the X-axis.
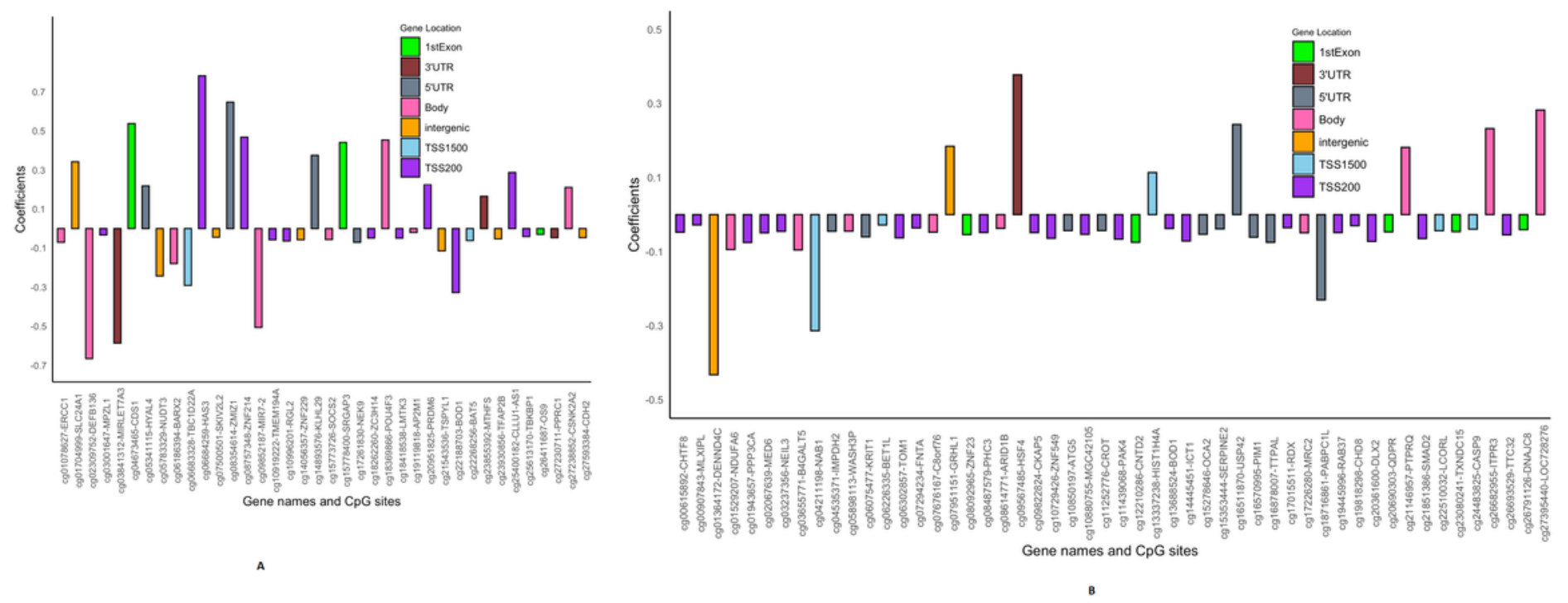

Figure 3

(A): Bar graph showing the main effects of DNAm on asthma acquisition at each of the 38 identified CpGs in IOWBC in males. Gene names corresponding to each $\mathrm{CpG}$ site are also labeled on the X-axis. (B): Bar graph showing the main effects of DNAm on asthma acquisition at each of the 52 identified CpGs in IOWBC in females. Gene names corresponding to each CpG site are also labeled on the X-axis. 


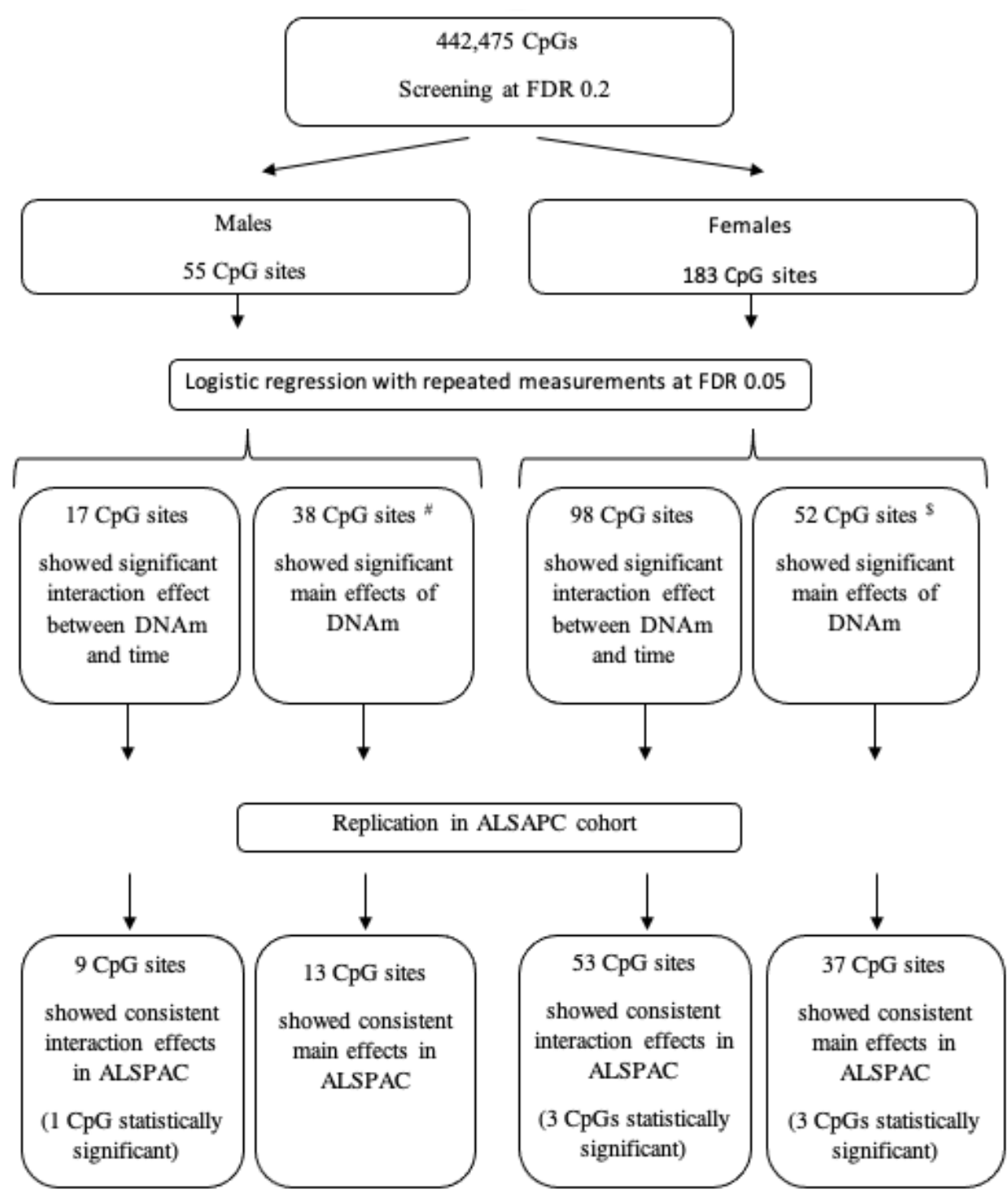

\section{Figure 4}

Flowchart of the study design and brief summaries of findings in each step. \# - Out of 38 CpGs after excluding significant interaction $\$=$ Out of $85 \mathrm{CpGs}$ after excluding significant interaction

\section{Supplementary Files}

This is a list of supplementary files associated with this preprint. Click to download.

- Tables.pdf

- Supplementtables.xlsx 